\title{
A Combination of Feature Extraction Methods with an Ensemble of Different Classifiers for Protein Structural Class Prediction Problem
}

\author{
Abdollah Dehzangi, Kuldip Paliwal, Alok Sharma, Omid Dehzangi, Abdul Sattar
}

\begin{abstract}
Better understanding of structural class of a given protein reveals important information about its overall folding type and its domain. It can also be directly used to provide critical information on general tertiary structure of a protein which has a profound impact on protein function determination and drug design. Despite tremendous enhancements made by pattern recognition-based approaches to solve this problem, it still remains as an unsolved issue for bioinformatics which demands more attention and exploration. In this study, we propose a novel feature extraction model which incorporates physicochemical and evolutionary-based information simultaneously. We also propose overlapped segmented distribution and autocorrelation based feature extraction methods to provide more local and global discriminatory information. The proposed feature extraction methods are explored for 15 most promising attributes that are selected from a wide range of physicochemical-based attributes. Finally, by applying an ensemble of different classifiers namely, Adaboost.M1, LogitBoost, Naive Bayes, Multi-Layer Perceptron (MLP), and Support Vector Machine (SVM) we show enhancement of the protein structural class prediction accuracy for four popular benchmarks.
\end{abstract}

Index Terms-Mixture of feature extraction models, overlapped segmented distribution, overlapped segmented autocorrelation, ensemble of different classifiers, physicochemical-based features

\section{INTRODUCTION}

Protein structural class prediction problem is defined as assigning a protein into one of the four well defined structural classes of proteins [1]. These structural classes are denoted by: all- $\alpha$, all- $\beta, \alpha+\beta$, and $\alpha / \beta$. The most accurate and popular structural classification of proteins can be found in Structural Classification of Proteins (SCOP) [2]. In the latest version of the SCOP, the number of structural classes has increased to 11 groups. However, these four major structural classes still cover almost $90 \%$ of proteins and are commonly used in many studies. In the biological perspective, protein structural class prediction problem is considered as an important task which provides crucial information about overall folding process and general functionality of the proteins. It also gives a better insight into protein fold recognition, protein secondary structure prediction and drug design [3], [4], [5]. Most of the approaches proposed in the literature to tackle this problem have been successfully applied to

- Abdollah Dehzangi is with Institute for Integrated and Intelligent Systems (IIIS), Griffith University, and National ICT Australia (NICTA), Brisbane, Australia. Email: a.dehzangi@griffith.edu.au

- Kuldip Paliwal is with School of Engineering, Griffith University, Brisbane, Australia. Email: k.paliwal@griffith.edu.au

- Alok Sharma is with School of Engineering, Griffith University, Brisbane, Australia and School of Engineering and Physics, University of the South Pacific, Fiji. Email: sharma_al@usp.ac.fj

- Omid Dehzangi is with Embedded System and Signal Processing Laboratory, Electrical Engineering Department, University of Texas at Dallas, Texas, United States of America. Email: omid.dehzangi@utdallas.edu

- Abdul Sattar is with Institute for Integrated and Intelligent Systems, Griffith University, and National ICT Australia (NICTA), Brisbane, Australia.Email: a.sattar@griffith.edu.au protein fold recognition and attained promising results [6], [7], [8].

From the pattern recognition perspective, this problem is presented as solving a multi-class classification task. The performance of the proposed method to solve this problem crucially relies on the selected attribute and consequently feature extraction method being used as well as the classification techniques being developed. During the past few decades a wide range of classification techniques such as, Meta-Classifiers [9], [10], [11], [12], Support Vector Machine (SVM) [13], [14], Artificial Neural Network (ANN) [15], [16], [17], and Ensemble classifiers [18], [19] have been used to tackle this problem. Among these classifiers, ensemble and SVM-based classifiers exhibited quite promising results [6], [7]. However, the performance of ensemble classifiers has not been adequately explored [20], [21], [22]. At the same time and in parallel by exploring the impact of classification techniques, a wide range of studies tried to tackle this problem by proposing novel feature extraction methods that maintain more local and global information for this task [3], [4], [5], [6], [23].

The most successful studies to enhance protein structural class prediction accuracy addressed both feature extraction and classification approaches simultaneously [6], [7], [18], [24]. However, it has been shown in the literature [3], [6], [7] that development of attribute selection and feature extraction methods tend to be more crucial for protein structural class prediction problem compared to the impact of classification technique being used. In general, features that have been used for this problem can be categorized into four groups namely, 
sequential, physicochemical, structural, and evolutionarybased features. The early studies conducted to tackle this problem have mostly relied on sequential-based (also called compositional-based) features which are extracted merely based on the alphabetic sequence of the amino acids [25]. Despite the importance of these features to provide significant discriminatory information based on the sequential similarities, they fail to perform properly when sequence similarity is low (which is also called twilight zone [3], [26]).

On the other hand, physicochemical-based features (extracted based on different physicochemical-based attributes (e.g. hydrophobicity and polarity) of the proteins and amino acids) are able to provide and maintain discriminatory information when that sequence similarity is low. Furthermore, these features are able to reveal the impact of different physicochemical-based attributes on the folding process. However, using just these group of features the protein structural class prediction accuracy remains limited. Note that in many abovereferenced studies, structural-based features (extracted based on predicted secondary structure of proteins (e.g. normalized frequency of $\alpha$-helix)) are categorized as physicochemical-based attributes [20], [27], [28]. Therefore, to maintain the consistency and for simplicity, these features are also referred as a kind of physicochemicalbased attributes in this study.

Recently, evolutionary-based features have been widely used for this problem and attained promising results [6], [29]. These features rely on the concept of substitution of the amino acids along the proteins through evolution and mainly extracted from the Position Specific Scoring Matrix (PSSM). PSSM is calculated by running PSIBLAST on a protein database [30]. The PSSM probabilities depend on the position of amino acids in the protein sequence. It provides important information about dynamic substitution score of an amino acid. Previously, PSSM was used to find transformed protein sequences (called consensus sequence) [3]. However, these features also suffer from the following two limitations: 1) it fails to maintain its discriminatory information especially when sequence similarity is low; and 2) it is unable to provide any information about the relation between physicochemical-based attributes and folding process [20].

To address these two limitations and at the same time to enhance the prediction performance, features derived from the predicted secondary structure using PSIPRED were used [3], [8], [31]. PSIPRED predicts protein secondary structure with about $80 \%$ prediction accuracy using the evolutionary-based information and PSIBLAST. However, due to this limited accuracy (about $80 \%$ ) of the predicted secondary structure by PSIPRED, it could not be relied as an adequate source of information for feature extraction to enhance the protein structural class prediction accuracy especially for over $80 \%$ [32]. It is also shown that studies relied on these features to enhance the prediction accuracy could not reach too far better results than $80 \%$ [3], [8], [31].

In this study, to address the above-mentioned limitations and to enhance the protein structural class prediction accuracy, a novel approach is proposed which comprises of both feature extraction and classification methods (with more consideration given to the attribute selection and feature extraction). The proposed method is implemented in the following four steps. In the first step, we conduct a comprehensive experimental study on a wide range of physicochemical-based attributes and select 15 most promising attributes which are explored using several previously reported feature extraction methods. In the second step, based on the concepts of overlapped segmented distribution and autocorrelation methods, two sets of features are extracted based on each attribute from the consensus sequence using evolutionary-based information. This approach enables us to obtain benefit of these two categories of features simultaneously to provide more local and global discriminatory information. In the third step, two sets of sequential-based features that attained good results in previous studies for this task are extracted and combined with our proposed features. In the final step, an ensemble of five different classifiers (AdaBoost.M1, LogitBoost, Naive Bayes, MLP, and SVM) is applied to the combination of the proposed features. The employed ensemble classifier was proposed in our previous work and attained promising results for the protein fold recognition [21]. However, it was not explored for the protein structural class prediction problem. The performance of the proposed approach is explored using four popular benchmarks. Our results show that the proposed approach in this study outperforms the protein structural class prediction accuracy compared to the results of previous studies for all of the employed benchmarks.

\section{Materials and Methodology}

\subsection{Benchmarks}

In this study, two popular benchmarks introduced by Zhou [25] are used to compare the performance of our proposed methods with the state-of-the-art methods found in the literature. The first benchmark denoted as Z277 consisting of 277 proteins and the second benchmark denoted as Z498 consisting of 498 proteins. Despite the relatively small size of these two benchmarks, they are still considered as important data sets for benchmarking. To explore the impact of the proposed methods on the low-similarity datasets, 1189 [33] and 25PDB [19] benchmarks which respectively consist of 1092 proteins with less than $25 \%$ sequential similarity and 1673 proteins with less than $40 \%$ sequential similarity are explored (Table 1).

\subsection{Explored Physicochemical-based Attributes}

In this study, 15 most promising physicochemical-based attributes which are taken from AAindex [34], APDbase 
TABLE 1

Explored benchmarks in this study.

\begin{tabular}{clccccc}
\hline Ref. & Benchmark & All- $\alpha$ & All- $\beta$ & $\alpha+\beta$ & $\alpha / \beta$ & Total \\
\hline$[25]$ & Z277 & 70 & 61 & 81 & 65 & 277 \\
{$[25]$} & Z498 & 107 & 126 & 136 & 129 & 498 \\
{$[33]$} & 1189 & 223 & 294 & 334 & 241 & 1092 \\
{$[19]$} & 25PDB & 443 & 443 & 346 & 441 & 1673 \\
\hline
\end{tabular}

[35], and previous studies found in the literature are explored [27]. We selected these 15 attributes among 115 physicochemical-based attributes which were experimentally explored using several classification techniques and feature extraction methods (mainly based on the overlapped segmented distribution and autocorrelationbased approaches). For a given attribute, we extracted six feature groups based on the overlapped segmented distribution and overlapped segmented autocorrelation approaches which are the subjects of this study. Then we applied five classifiers namely, Adaboost.M1, Random Forest, Naive Bayes, K-Nearest Neighbor (KNN), and SVM to each feature group separately. Therefore, 30 prediction accuracies were achieved for each physicochemicalbased attribute (five classifiers applied to six feature groups separately $(5 \times 6=30))$. Then we compared these results for all 115 attributes and selected 15 attributes that attained the best results in average for all 30 prediction accuracies. The attribute selection process is explained in detail in Appendix A. Explored attributes in this study are namely, (1) structure derived hydrophobicity value, (2) polarity, (3) average long range contact energy, (4) average medium range contact energy, (5) mean Root Mean Square (RMS) fluctuational displacement, (6) total non-bounded contact energy, (7) amino acids partition energy, (8) normalized frequency of $\alpha$-helix, (9) normalized frequency of $\beta$ turns, (10) hydrophobicity scale derived from 3D data, (11) hydrophilicity scale derived from High-Performance Liquid Chromatography (HPLC) peptide retention data ${ }^{1}$, (12) average gain ratio of surrounding hydrophobicity, (13) mean fractional area loss, (14) flexibility, and (15) bulkiness.

Most of the selected attributes have not been adequately (or not at all) explored for the protein structural class prediction problem (or for the protein fold recognition [3], [20], [28]); while in our experimental study, they outperformed even the popular attributes that had been widely used to tackle this task.

\subsection{Feature Extraction Approach}

In this study, we concatenate features driven from the all three main sources (sequential, physicochemical and evolutionary-based features) to form a feature vector which is used for the protein structural class prediction problem. In the first step, PSSM is calculated by applying the PSIBLAST on NCBI's non redundant (NR)

1. This attribute is used to predict which areas of a protein are on the surface data base for our explored benchmarks (cut off value (E) set to 0.001) [3], [6], [36]. The PSSM consists of two $L \times 20$ matrices ( $L$ is the length of a protein and the columns of the matrices represent 20 amino acids) [6]. The first matrix is called PSSM_cons and gives the log-odd of the substitution score. The second matrix is called PSSM_prob and gives the normalized probability of substitution score for each amino acid. In the second step, two important sequential-based feature sets are extracted from the PSSM. In the third step, consensus sequence is extracted directly from the PSSM and then, physicochemical-based features are extracted from this sequence instead of using the original sequence (as it was used conventionally). In the next step, extracted features are combined with the extracted features in the previous steps. In this way, the true potential of all the three categories of attributes are considered and explored. In continuation, each approach will be explained in detail.

\subsubsection{Sequential-based Feature Extraction from Evolutionary-based Information}

To explore the impact of the sequential-based features, two feature groups namely, evolutionary-based composition (PSSM_AAC) and evolutionary-based auto covariance (PSSM_AC) are extracted from the PSSM matrix. These features capture significant local and global information and have been used in the past [3], [6], [36] with promising results for the protein structural class prediction problem.

2.3.1.1 Evolutionary-based Composition Feature Group (PSSM_AAC): This feature group is extracted based on the concept of composition of the amino acids feature group to provide discriminatory information related to the occurrence of each amino acid along a given protein sequence [6]. The difference between the PSSM_AAC and the composition features derived from the original protein sequence (which is extracted by counting the occurrence of each amino acids along the protein sequence divided by the length of the protein) is that the PSSM_AAC is extracted from the PSSM_cons by summing the substitution score of each amino acids and divide it by the total length of the protein.

$$
P S S M_{-} A A C_{j}=\frac{1}{L} \sum_{i=1}^{L} S_{i j}, \quad(j=1, \ldots, 20),
$$

where $L$ is the length of protein and $S_{i j}$ is the substitution score of the amino acids at location $i$ by $j-t h$ amino acid in the PSSM_cons.

2.3.1.2 Evolutionary-based Auto Covariance Feature Group (PSSM_AC): To provide more information about the interaction of the amino acids with each other along a protein sequence, the concept of PSSM_AC is used recently in the literature [6], [36]. PSSM_AC gives the auto covariance of the substitution score of each amino acid along a protein sequence and is defined as follows: 


$$
\begin{gathered}
P S S M_{-} A C_{k, j}=\frac{1}{(L-k)} \sum_{i=1}^{L-k}\left(S_{i, j}-S_{a v e, j}\right)\left(S_{i+k, j}-S_{a v e, j}\right), \\
\left(j=1, \ldots 20 \text { and } k=1, \ldots, F_{s}\right),
\end{gathered}
$$

where $S_{a v e, j}$ is the average of substitution score of the amino acid $i$ in the PSSM_cons and $F_{s}$ is the distance factor. Therefore, in total $20 \times F_{s}$ features are calculated in this feature group. In this study, two values of the distance factor $\left(F_{s}=6\right.$ or 10$)$ are investigated as they were presented in the literature as the most effective distance factors for protein structural class and protein fold prediction problems [6], [36]. Note that the combination of these two feature groups PSSM_AAC, and PSSM_AC is called AAC-PSSM-AC in the literature (which will also be referred similarly for the rest of this study).

\subsubsection{Consensus Sequence Extraction Method}

Consensus sequence is extracted to reveal more evolutionary information considering the PSSM compared to the original protein sequence. It was also shown as an effective way to provide more information for the protein structural class prediction problem [3], [7], [18]. To extract the consensus sequence, previous studies replaced the amino acid at a given location in the original protein sequence by the amino acid with the maximum substitution score in the row corresponding to that location in the PSSM. This is done using the following two steps. In the first step, the index is found as:

$$
I_{i}=\operatorname{argmax}\left\{S_{i j}: 1 \leq j \leq 20\right\}, 1 \leq i \leq L,
$$

where $S_{i j}$ is the substitution score of the amino acid at location $i$ with the $j$-th amino acid in the PSSM_cons. In the second step, replace the amino acid at $i-t h$ location of original protein sequence by the $I_{i}-t h$ amino acid to form the consensus sequence $C_{1}, C_{2}, \ldots, C_{L}$. However, due to the fact that the PSSM_cons consists of the logodds of the substitution score, in many cases there are more than one maximum for the substitution score in this matrix. In this case, the selection of the better value from multiple maximum values were conducted randomly (the first/last maximum values) which can not be considered as an accurate method. Furthermore, the PSSM_cons does not provide any beneficial information about unknown amino acids in proteins (which are conventionally shown by ' $X$ ' in the sequence). The substitution scores for these unknown amino acids in the PSSM_cons are all equal to - 1 . Therefore, relying merely on the PSSM_cons left the issue of unknown proteins unaddressed.

By exploring the PSSM_prob in more detail, we realized that the occurrence of multiple maximum is much less frequent in this matrix compared to the PSSM_cons due to the fact that it returns the normalized probability of substitution score (due to better precision). In addition, in case that a similar sequence is spotted in the non-redundant protein data bank, this matrix provides substitution score probability even for unknown amino acids (if none is spotted it returns zero which rarely occurs just in case that the length of the protein is very short and at the same time unknown amino acids are spotted). Therefore, it is possible to address the issue of unknown amino acids using evolutionary-based information and considering the PSSM_prob.

Hence, in this study, we propose a novel consensus sequence extraction model for the protein structural class prediction problem considering the concepts of the PSSM_prob and the PSSM_cons simultaneously. In our method, we first check the PSSM_prob. In case that a unique maximum is spotted, it will be replaced with the original amino acids in the sequence. Otherwise, we will refer to the PSSM_cons to find the maximum. If a unique maximum is spotted in this matrix, it will be replaced with the original amino acids sequence. Otherwise, the first maximum from the PSSM_prob will be replaced. The most crucial impact of the explored method in this study is proposing a method to address the issue of unknown amino acids. In this method, unknown amino acids are transformed to the consensus sequence considering the evolutionary-based information which are extracted from the PSSM_prob. Our approach successfully addresses the issue of unknown amino acids for the explored benchmarks. Using our proposed consensus sequence extraction method, for 25PDB and 1189 benchmarks, all unknown amino acids are replaced and for Z277 and Z498 all but one protein, unknown amino acids are replaced.

\subsubsection{Physicochemical-based Feature Extraction Method}

To explore the potential of the physicochemical-based attributes for the protein structural class prediction problem better, a novel feature extraction model based on the concept of the evolutionary-based information is proposed. In the proposed model, we first transform the original protein sequence to its consensus sequence as described before. Then from the consensus sequence, physicochemical-based features are extracted using overlapped segmented distribution and autocorrelation-based methods which are proposed in this study. Proposed approaches are aimed at providing more local and global discriminatory information.

As highlighted earlier, previous approaches mainly relied on the original protein sequence to extract the physicochemical-based features [3], [4]. In our approach, we use the consensus sequence $\left(C_{1}, C_{2}, \ldots, C_{L}\right)$ to derive a numerical sequence $R_{1}, R_{2}, \ldots, R_{L}$ where $R_{i}$ is the numerical value of a particular physicochemical attribute (e.g. polarity) of amino acid $C_{i}$. This sequence is then used by overlapped segmented distribution and autocorrelation-based methods to derive features. In this manner, we are able to explore the discriminatory information (shown during the experimentation) provided by the evolutionary and physicochemical-based attributes simultaneously which not only enhances the protein 


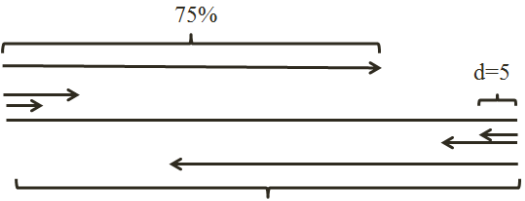

All

Fig. 1. Overlapped segmented distribution-based feature extraction method.

structural class prediction accuracy but it also enables us to provide crucial information about the impact of a given physicochemical-based attribute on the folding process. The overlapped segmented distribution and autocorrelation methods are explained below in detail.

2.3.3.1 Overlapped Segmented Distribution Approach: Global density of different attributes is widely used in protein science and believed to provide important information of the global impact of a specific attribute on the folding process (e.g. polarity) [3], [27]. It is defined as follows:

$$
T_{\text {global_density }}=\frac{\sum_{i=1}^{L} R_{i}}{L},
$$

where $R_{i}$ is the attribute value (normalized) of the $i$-th amino acid. However, it fails to provide adequate local information [37]. Therefore, global density is not considered as an effective feature to appropriately explore the potential discriminatory information of a given attribute. In this study, instead of relying solely on the global density of a given attribute, we also use the distribution of the amino acids based on the segmented density.

In the proposed approach, we first compute $T=$ $T_{\text {global_density }} \times L$, which is the total sum of the attribute sequence $R_{i}(i=1, \ldots, L)$. In the second step, starting from left side of the attribute sequence, we find the index I such that the partial sum $S=R_{1}+R_{2}+\ldots+R_{i}$ of the first $I_{1}^{(f)}$ (in which the superscript $f$ stands for starting from the left side of the proteins) attributes reaches to $d \%$ (called distribution factor) of $T$ (i.e., $S \geq(T \times d) / 100$ ). This process is carried out for different values of $d(5 \%$, $10 \%, 15 \%, \ldots, 75 \%)$ to get 15 indices $I_{1}^{(f)}, I_{2}^{(f)}, \ldots, I_{15}^{(f)}$. These indices are divided by the length of protein to obtain 15 distribution features. Note that these 15 features are obtained by analyzing the sequence in the forward direction (i.e., starting from the left). We also compute 15 features by analyzing the sequence in the backward direction (i.e., starting from the right). Thus, a total of 31 features using the proposed method are extracted (1 global density +15 from the left side +15 from the right side). Note that we segment the protein sequence with distribution factor of $d$ and process it from the left as well as from the right side of the protein sequence while the left and right side processing are having overlap (Figure 1). As a result, we call this method overlapped segmented distribution approach.

In this study, $5 \%$ distribution factor and $75 \%$ called overlapping factor, are selected based on the average length of the proteins in the explored benchmarks which is the trade off between the number of produced features and the time consumption of this task, and experimental study that was conducted by the authors. The overlapping approaches are proposed to provide more information about the distribution of the amino acids in the middle of a protein considering each side. Considering the number of features (only 10 overlap features), this approach is able to provide crucial overlapping information to tackle this problem.

This approach also enables us to explore the impact of each attribute more comprehensively compared to previously explored methods [3], [20], [28], [38], [39]. It is important to highlight that due to use of density, the segmentation factor is independent from the length. It makes our method more appropriate for general cases where the gap between the length of the shortest and the longest proteins in the data bank is large compared to previously used distribution-based approaches [28].

2.3.3.2 Overlapped Segmented Autocorrelation: In the past, the autocorrelation features have been computed using the whole protein sequence of $L$ attribute values $R_{i}(i=1, \ldots, L)$. Pseudo amino acid composition based features are good examples of these type of features [39]. These autocorrelation features capture the interaction of the neighboring amino acids over the entire length of the protein sequence. In the present study, we extend the concept of overlapped segmented distribution features as described in the previous subsection to compute the autocorrelation features from the segmented protein sequence. This is done to provide more local discriminatory information. Here we segment the protein sequence using distribution factor of $10 \%$ $(d=10)$ and overlapping factor of $70 \%\left(o_{f}=70\right)$. Using a procedure similar to the one described in the previous subsection, we first analyze the protein sequence in forward direction and find seven indices $I_{1}^{(f)}, I_{2}^{(f)}, \ldots, I_{7}^{(f)}$ for seven different values of $d(d=10 \%, 20 \%, \ldots, 70 \%)$. These seven indices are used to segment the protein sequence into seven segments and $F_{p h}$ number of autocorrelation coefficients for each of these segments are computed as follows:

$$
\begin{aligned}
\text { Autocorrelation }_{i, k}=\frac{1}{\left(I_{k}^{(f)}-i\right)} \sum_{j=1}^{I_{k}^{(f)}-i} R_{j} R_{j+i}, \\
\quad\left(k=1,2, \ldots, 7 \text { and } i=1, \ldots, F_{p h}\right),
\end{aligned}
$$

Note that $7 \times F_{p h}$ autocorrelation coefficients are computed in this manner by analyzing the protein sequence in the forward direction. This process is repeated to obtain another $7 \times F_{p h}$ autocorrelation coefficients by analyzing the protein sequence in the backward direction. We also compute the global autocorrelation coefficient of the whole protein sequence to provide more global information in conjunction with the extracted local information. Thus, we have extracted a total of 
$\left(7 F_{p h}+7 F_{p h}+F_{p h}\right)=15 F_{p h}$ autocorrelation features in this manner. Two values of $F_{p h}(6$ and 10) are investigated in this study; which gives $90(42+42+6)$ features for $F_{p h}=6$ and $150(70+70+10)$ features for $F_{p h}=10$.

Finally, the extracted feature groups based on both physicochemical-based feature extraction methods for a given attribute are combined to provide local and global discriminatory information based on density, distribution, and autocorrelation approaches simultaneously. Therefore, considering two different autocorrelation distance factors, two combined feature groups with 121 and 181 features are produced for $F_{p h}=6$ and $F_{p h}=10$, respectively.

\subsection{Ensemble of Different Classifiers}

Instead of using a single classifier, we use an ensemble of different classifiers for protein structural class prediction task. A well-defined ensemble of these classifiers is capable of addressing statistical, computational, and representational issues better than an individual classifier [19], [21], [40].

For an ensemble classifier, diversity and individual accuracy of its component classifiers are two main criteria that define its classification performance [41]. In an ensemble classifier, diversity is encouraged by exploring classifiers that have diverse learning policies (or diversely trained) and individual accuracy is encouraged by the individual performance of the explored classifiers for an specific task.

In our previous work, we proposed an ensemble of five different classifiers namely, AdaBoost.M1, LogitBoost, Naive Bayes, SVM, and MLP that was successfully explored for the protein fold recognition and attained promising results [21], [42]. These five classifiers also have been explored individually for protein structural class prediction problem in different studies and attained promising results (which encourage individual accuracy of the ensemble classifier built base on these classifiers [5], [10], [16], [33]). Using these five classifiers also encourage diversity due to their different learning and classification approaches which are studied in [21], [42]. In the present study, we employ the same five classifiers to construct an ensemble classifier used for the classification task. Note that the implementations of these classifiers (AdaBoost.M1, LogitBoost, MLP, SVM, and Naive Bayes) in WEKA machine learning toolbox are used [43]. To combine the employed classifiers, we use majority voting which attained better results compared to other explored algebraic combiners. We describe briefly each of these individual classifiers below.

\subsubsection{AdaBoost.M1}

AdaBoost.M1 was introduced by [44] based on boosting approach. AdaBoost.M1 sequentially applies a base learner to bootstrap samples of data and adjusts the weight of the misclassified samples in each iteration to minimize the exponential loss function. In the final step, by combining all classifiers in each iteration using majority voting, it creates a diverse classifier. AdaBoost.M1 is considered as the best-of-the-shelf Metaclassifiers which attained promising results for a wide range of applications as well as protein structural class prediction problem [10], [45]. In this study, Adaboost.M1 using C4.5 decision tree as its base learner is used with 100 iterations; these many iterations have been found to provide the best results for similar studies in the past [20].

\subsubsection{LogitBoost}

Similar to the Adaboost.M1, LogitBoost is considered as a kind of Meta-classifier based on boosting approach [46]. It was introduced to address the over fitting problem in AdaBoost.M1 which arises when the number of training samples is small. In this classifier, logistic regression function is employed as a base learner and in each iteration it minimizes logistic loss function to improve the performance of its base learner. Similar to the Adaboost.M1, this classifier has been widely used in protein science and attained promising results [9], [12], [20]. In this study, the number of iterations for this classifier is set to 100 (which attained the best results for similar studies [20].).

\subsubsection{Support Vector Machine (SVM)}

This classifier is considered as the-state-of-the-art classifier in the pattern recognition as it outperforms other individual classifiers in tackling this problem [6]. SVM aims at minimizing the prediction error by finding the Maximal Marginal Hyperplane ( $M M H)$ based on the support vector theory [47]. It transforms the input data using kernel trick to find appropriate support vectors to achieve its goals. We use SVM using Sequential Minimal Optimization (SMO) algorithm with polynomial kernel of degree one (which is called linear kernel) to reduce the time complexity of our proposed classifier ${ }^{2}$. It was also shown that using linear kernel attained similar results compared to use of kernel degree of two and three as a part of proposed ensemble classifier. For this classifier, the regularization parameter is set to four as the number of classes for protein structural class prediction problem.

\subsubsection{Multi-Layer Perceptron (MLP)}

It is considered as one the most popular Artificial Neural Network (ANN) based classifiers [21]. It uses gradient descent in its interconnected network in the feedforward method to minimize the prediction error function over

2. We have studied a number of kernels such as linear, polynomial, Gaussian, and Radial Basis Function (RBF) for the SVM classifier and used this classifier on its own (i.e., without putting it in the ensemble configuration) and found the polynomial kernel (with polynomial degree $p=3$ ) giving the best results. When we studied these kernels for the SVM classifiers when this classifier is used in the ensemble configuration, we found all the kernels are similar in terms of their performance. Since linear kernel is simple and does not need much tuning, we provide in this paper the results of the ensemble configuration with the SVM classifier using linear kernel. 


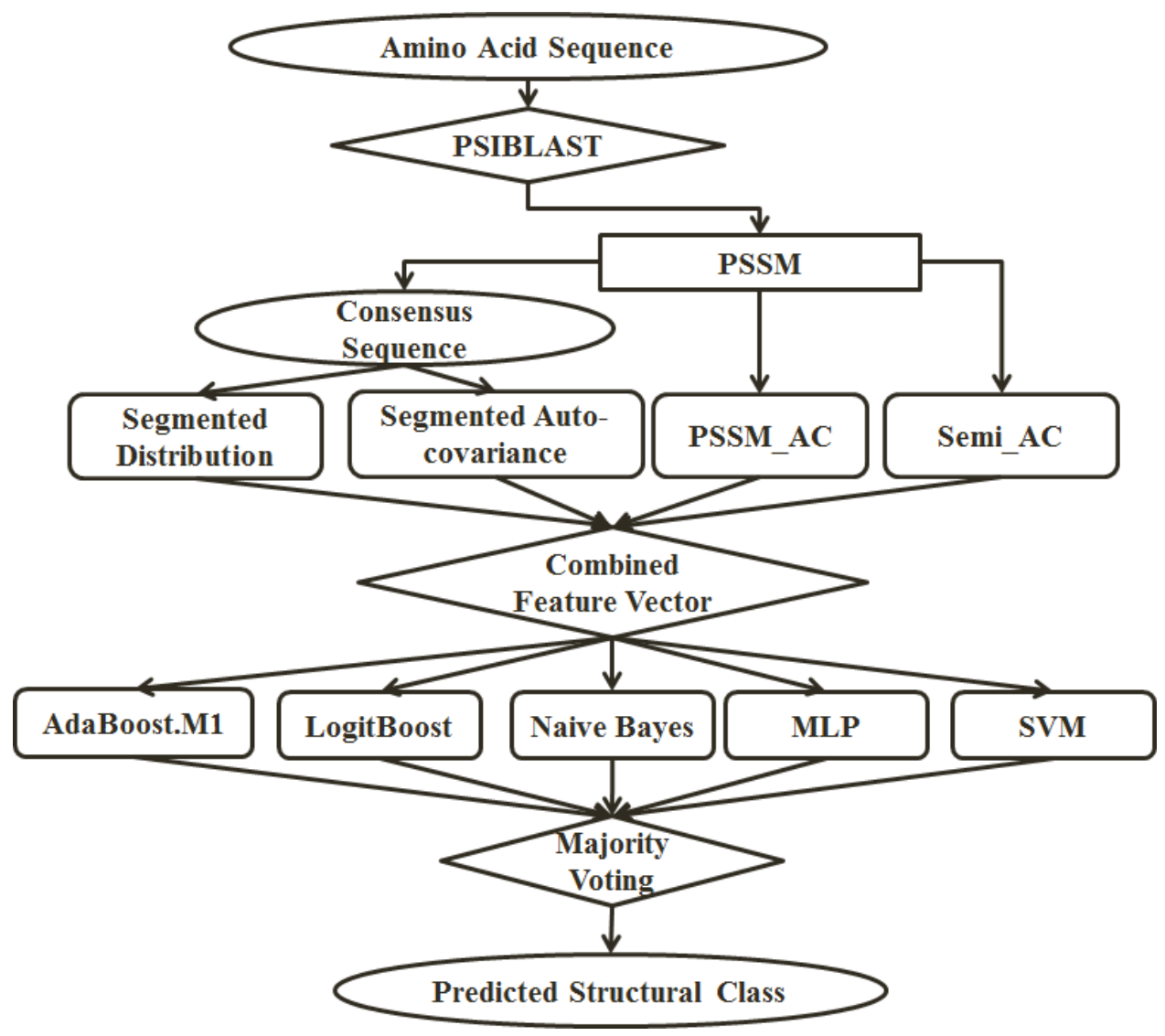

Fig. 2. The overall architecture of the proposed method.

the training data. Despite its simplicity compared to the other ANN-based classifiers, it has achieved comparable results (and sometimes even better results) for similar studies [15], [20], [48]. In this study, the default parameters of WEKA used for MLP are adopted (number of hidden layers is set to one and number of hidden nodes where set to number of input features).

\subsubsection{Naive Bayes}

This classifier assumes the independence of features which helps in computing the posterior probability required in the Bayes rule in a simple manner [21], [33]. Despite its naive assumption, it has been popularly used for different tasks and attained promising results for similar studies found in the literature [49]. Naive Bayes classifier uses the features in a manner different from other classifiers and hence, when it is used as a component of an ensemble classifier it has attained good results for the protein fold classification task [42].

\section{Results And Discussion}

As mentioned in section 2, we propose in this paper a feature extraction method and ensemble of different classifiers for protein structural class prediction problem. The feature extraction method uses individual physicochemical-based attributes to extract the following two kinds of features: 1) segmented distribution, and 2) segmented autocorrelation. In this section, we investigate these two kinds of features for each of the 15 physicochemical-based attributes. These features are concatenated with PSSM_AAC and PSSM_AC features to form the final feature vector used for classification task.

Note that most of the studies conducted on the Z277 and Z498 benchmarks used Jackknife evaluation criterion while studies conducted on the 1189 and 25PDB benchmarks used 10-fold cross validation evaluation criterion. In this study, we use 10-fold cross validation to report our results on the four benchmarks (Z277, Z498, 1189, and 25PDB). As it was shown in [50], crossvalidation evaluation criterion produces similar results compared to the use of Jackknife method (while using cross-validation produces slightly less biased results specially when the number of samples increases). To the best of our knowledge, the results reported by Liu and his coworkers using SVM classifier with Radial Based Function (RBF) kernel (implemented in the SVMLIB) are the best results for these four benchmarks [6].

We use ensemble of different classifiers to classify the feature vector introduced earlier. The general architecture of the proposed method is shown in Figure 2. For the rest of this study, the combination of the feature groups based on each attribute and considering $F_{p h}$ (distance factor used in overlapped segmented au- 
tocorrelation feature group) and $F_{s}$ (distance factor used in evolutionary-based auto covariance feature group) will be shown by: (comb_num, $F_{p h}, F_{s}$ ). To be able to compare our results with previous studies found in the literature, we report our results in terms of protein structural class prediction (or classification) accuracy (in percentage) which is defined as follows:

$$
Q=\frac{C}{N} \times 100,
$$

where $C$ is the number of the correctly classified test samples and $N$ is the total number of test samples.

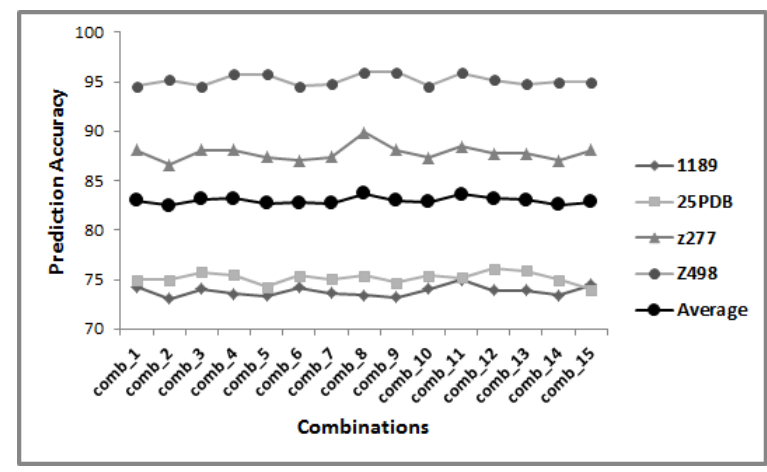

Fig. 3. The prediction accuracies achieved (in percentage) for applying ensemble of classifiers to 15 combinations of feature vectors using $F_{p h}=6$ and $F_{s}=6$ for feature extraction.

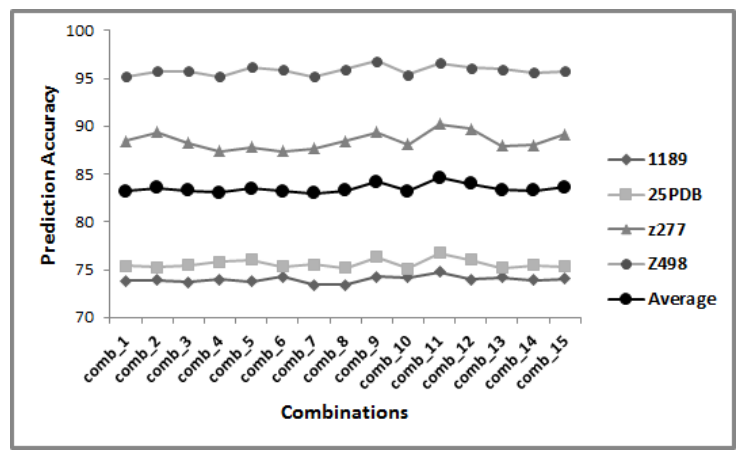

Fig. 4. The prediction accuracies achieved (in percentage) for applying ensemble of classifiers to 15 combinations of feature vectors using $F_{p h}=10$ and $F_{s}=6$ for feature extraction.

\subsection{The Impact of $F_{p h}$ and $F_{s}$ on the Prediction Performance}

In this subsection, we use PSSM_AAC, PSSM_AC, overlapped segmented distribution, and overlapped segmented autocorrelation feature groups and investigate the impact of $F_{p h}$ and $F_{s}$ on the protein structural class prediction problem. This is done to arrive at the best combination of $F_{p h}$ and $F_{s}$ values. With $F_{p h}=6$ or 10 and $F_{s}=6$ or 10 , the following four combinations of $F_{p h}$ and $F_{s}$ are possible: 1) $\left.F_{p h}=6, F_{s}=6,2\right) F_{p h}$

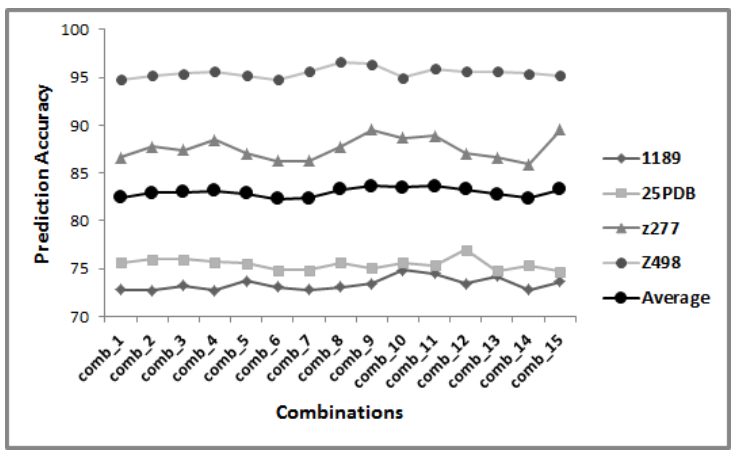

Fig. 5. The prediction accuracies achieved (in percentage) for applying ensemble of classifiers to 15 combinations of feature vectors using $F_{p h}=10$ and $F_{s}=10$ for feature extraction.

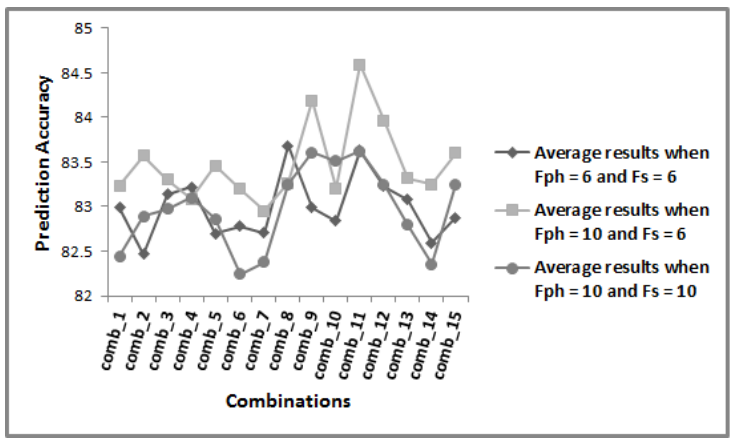

Fig. 6. The comparison of the prediction accuracies driven from the average results (in percentage) achieved by adjusting $F_{p h}=6$ and $F_{s}=6, F_{p h}=10$ and $F_{s}=6$, and $F_{p h}=10$ and $F_{s}=10$ for all 15 combinations of feature vectors.

$\left.=6, F_{s}=10,3\right) F_{p h}=10, F_{s}=6$, and 4) $F_{p h}=$ $10, F_{s}=10$. Since the combination $\mathrm{Fph}=6$ and $\mathrm{Fs}=10$ gives results similar to combination $\mathrm{Fph}=6$ and $F_{s}=6$ in our experimental study, we have not shown it here to keep the paper as brief as possible. We apply our proposed ensemble of classifiers to all the combinations of feature vectors extracted in this study with respect to the values of $F_{p h}$ and $F_{s}$. The results achieved by applying the ensemble classifier (which is an ensemble of five classifiers as described in previous section) to 15 combinations of features extracted for $F_{p h}=6$ and $F_{s}=$ 6 (comb_num,6,6); $F_{p h}=10$ and $F_{s}=6$ (comb_num,10,6); and $F_{p h}=10$ and $F_{s}=10($ comb_num, 10,10) for all benchmarks and their average prediction accuracies (for each combination) are shown in Figure 3, Figure 4, and Figure 5, respectively.

In Figure 6, the average prediction accuracies achieved for all 15 combinations of feature vectors for all four benchmarks investigated in this study by adjusting $F_{p h}=6$ and $F_{s}=6, F_{p h}=10$ and $F_{s}=6$, and $F_{p h}=10$ and $F_{s}=10$ are shown and compared. As we can see, the average results achieved by adopting $F_{p h}=10$ and $F_{s}=$ 6 attains relatively better results then two other alternatives used for $F_{p h}$ and $F_{s}$. Therefore, we adopt $F_{p h}=10$ 
and $F_{s}=6$ as the most effective values for these two parameters and the report the results in this paper from here onwards using these values. Note that the highest results in average for all these four benchmarks achieved by using physicochemical-based attributes number 9,11 , and 12 (normalized frequency of $\beta$-turn, hydrophilicity scale derived from (HPLC) peptide retention data, and average gain ratio of surrounding hydrophobicity, respectively) which to the best of our knowledge have not been adequately explored for feature extraction for the protein structural class prediction problem in the literature.

These three attributes are based on hydrophobicity and predicted secondary structure of the proteins which are considered as the most important aspect of proteins and amino acids that impact on the folding process in the biological perspective. Achieving best results using hydrophobicity and predicted secondary structure of proteins also highlights their effectiveness in the computational aspect which can be explored using segmentationbased feature extraction method. Furthermore, the better performance attained by using these three attributes highlight their effectiveness and preference compared to use of the other, more popular, attributes to enumerate hydrophobicity and predicted secondary structure of proteins (e.g. structure derived hydrophobicity value, normalized frequency of alpha-helix (which both are also investigated in this study (attribute numbers 1 and 8 respectively)). Among these three physicochemicalbased attributes, attribute number 11 attained the best results in average for all four benchmarks explored in this study.

\subsection{The Impact of Proposed Features Versus Classi- fication Technique Used in This Study}

In this subsection, we separately investigate the impact of proposed feature extraction techniques and the ensemble classifier on the protein structural class prediction accuracy. To do this, we start with the study reported by Liu and his co-workers as it has reported the best results for this prediction problem as mentioned earlier [6]. They have used SVM classifier with the combination of PSSM_AAC and PSSM_AC feature groups where $F_{s}$ is set to 6 (140 features in total) and called this feature combination as AAC-PSSM-AC. In this subsection, we use the same SVM classifier with the same AAC-PSSM-AC features and report the protein structural class prediction results in the first row of Table 2 for the four benchmarks described earlier. These results are used here as baseline results which we want to improve by using our feature extraction techniques and the ensemble classifier. For this, we first study the impact of adding the overlapped segment distribution feature group to the baseline features (AAC-PSSM-AC) and use the SVM classifier to obtain the results as shown in the second row of Table 2.

Results with the overlapped segmented autocorrelation feature group added to the baseline feature are shown in the third row. These results clearly indicate that overlapped segmented feature group as well as overlapped segmented autocorrelation feature group help individually to improve the results, though the later group does slightly better. When both of these groups are added to the baseline features, the results (shown in the forth row) become much better. Finally, we use the ensemble classifier (instead of SVM classifier) with both the feature groups added to the baseline features and results are shown in the fifth row of Table 2. These results are better than the results listed in the fourth row indicating the improvement in performance resulting from the ensemble classifier over the SVM classifier. We can also see from this table that these results (in the fifth row) are better than the baseline results demonstrating the importance of our feature extraction method and the ensemble classifier.

In order to ascertain the statistical significance of this improvement, we use paired t-test and find $t$-value equal to 6.7293 with 3 degrees of freedom. These results are statistically better than the baseline results at $5 \%$ significance level ( $p$ value equals to 0.0034 ). This shows the significance of our proposed feature extraction and ensemble classifier with respect to baseline results.

\subsection{Reported Results in This Study Compared to the Results Reported in the Literature}

In this section, we use AAC-PSSM-AC, overlapped segmented distribution, and overlapped segmented autocorrelation as features with the ensemble classifier for protein structural class prediction problem. The resulting prediction accuracy (in percentage) is shown in Tables 3, 4, 5, and 6 for the Z277, Z498, 1189, and the 25PDB benchmarks, respectively, for the three physicochemicalbased attributes (attributes number 9, 11, 12) using $F_{p h}=10$ and $F_{s}=6$ (comb_9,10,6, comb_11,10,6, and comb_12,10,6). For comparison, we also list the best results found in the previous studies reported in the literature $^{3}$. For each benchmark, the prediction performance for each structural class is also reported separately in these tables.

As shown in Table 3, we achieve over $90 \%$ prediction accuracy for the Z277 benchmark, which is $1.5 \%$ better than the highest result that was reported for this benchmark before. By reproducing the result of [6] on this benchmark (using 10 -fold cross validation) $88.8 \%$ prediction accuracy is achieved while for our method, we reach up to $90.3 \%$ (for Comb_11,10,6) prediction accuracy. Similarly, we achieve $96.8 \%$ prediction accuracy using (Comb_9,10,6) feature set for the Z498 benchmark (Table 4), which is better than the best prediction accuracy

3. As highlighted earlier in Section 1, the prediction accuracy of the PSIPRED for the protein secondary structure prediction is about $80 \%$. Therefore, as discussed in [32], it can not be considered as a reliable feature source as it could not contribute to enhance the protein structural class prediction accuracy too far better than $80 \%$. Therefore, these tables show results from previous studies without the use of PSIPRED [3], [8], [31]). 
TABLE 2

The impact of proposed feature extraction groups versus ensemble of classifiers proposed in this study to enhance protein structural class prediction accuracy for attribute number 11 where $F_{p h}$ and $F_{s}$ are respectively set to 10 and 6 .

\begin{tabular}{|c|c|c|c|c|c|c|}
\hline Combination of features & Classifier & Z277 & Z498 & 25DDB & 1189 & Average \\
\hline AAC-PSSM-AC & SVM & 88.8 & 95.0 & 73.9 & 72.7 & 82.6 \\
\hline AAC-PSSM-AC + overlapped segmented distribution & SVM & 88.9 & 95.3 & 74.4 & 73.0 & 82.9 \\
\hline AAC-PSSM-AC + overlapped segmented autocorrelation & SVM & 89.2 & 95.5 & 75.3 & 73.8 & 83.5 \\
\hline AAC-PSSM-AC + overlapped segmented distribution + overlapped segmented autocorrelation & SVM & 89.7 & 96.1 & 75.8 & 74.2 & 83.9 \\
\hline AAC-PSSM-AC + overlapped segmented distribution + overlapped segmented autocorrelation & Ensemble Classifier & 90.3 & 96.6 & 76.7 & 74.8 & 84.6 \\
\hline
\end{tabular}

TABLE 3

The best results (in percentage \%) achieved for Z277 benchmark (for combinations of feature vectors extracted for attributes number 9,10 , and 12 while $F_{p h}$ and $F_{s}$ are respectively set to 10 and 6) compared to the best results reported in the literature

\begin{tabular}{llccccc}
\hline References & Method & All- $\alpha$ & All- $\beta$ & $\alpha+\beta$ & $\alpha / \beta$ & Overall \\
\hline$[15]$ & Neural Network & 68.6 & 85.2 & 86.4 & 56.9 & 74.7 \\
{$[25]$} & Component Coupled & 84.3 & 82.0 & 81.5 & 67.7 & 79.1 \\
{$[51]$} & SVM & 74.3 & 82.0 & 87.7 & 72.3 & 79.4 \\
{$[9]$} & LogitBoost & 81.4 & 88.5 & 92.6 & 72.3 & 84.1 \\
{$[4]$} & IGA-SVM & 84.3 & 85.5 & 92.6 & 70.7 & 84.5 \\
{$[52]$} & CWT-PCA-SVM & 85.7 & 90.2 & 87.7 & 80.1 & 85.9 \\
{$[18]$} & IB1 & 89.7 & 88.1 & 92.2 & 80.0 & 87.7 \\
{$[39]$} & SVM Fusion & 85.7 & 90.2 & 93.8 & 80.0 & 87.7 \\
{$[6]$} & AAC-PSSM-AC & 86.2 & 92.9 & 95.0 & 80.3 & 88.8 \\
This Study & Comb_9,10,6 & 88.4 & 91.8 & 95.1 & 81.3 & 89.4 \\
This Study & Comb_11,10,6 & 90.0 & 93.4 & 96.3 & 80.0 & $\mathbf{9 0 . 3}$ \\
This Study & Comb_12,10,6 & 88.7 & 95.1 & 93.8 & 80.2 & 89.7 \\
\hline
\end{tabular}

TABLE 4

The best results (in percentage \%) achieved for Z498 benchmark (for combinations of feature vectors extracted for attributes number 9,10 , and 12 while $F_{p h}$ and $F_{s}$ are respectively set to 10 and 6 ) compared to the best results reported in the literature.

\begin{tabular}{llccccc}
\hline References & Method & All- $\alpha$ & All- $\beta$ & $\alpha+\beta$ & $\alpha / \beta$ & Overall \\
\hline$[15]$ & Neural Network & 86.0 & 96.0 & 88.2 & 86.0 & 89.2 \\
{$[25]$} & Component Coupled & 93.5 & 88.9 & 90.4 & 84.5 & 89.2 \\
{$[39]$} & SVM Fusion & 99.1 & 96.0 & 80.9 & 91.5 & 91.4 \\
{$[51]$} & SVM & 88.8 & 95.2 & 96.3 & 91.5 & 93.2 \\
{$[4]$} & IGA-SVM & 96.3 & 93.6 & 97.8 & 89.2 & 94.2 \\
{$[9]$} & LogitBoost & 92.6 & 96.0 & 97.1 & 93.0 & 94.8 \\
{$[6]$} & AAC-PSSM-AC & 94.0 & 96.0 & 97.1 & 92.5 & 95.0 \\
{$[52]$} & CWT-PCA-SVM & 94.4 & 96.8 & 97.0 & 92.3 & 95.2 \\
{$[18]$} & IB1 & 95.0 & 95.8 & 97.8 & 94.2 & 95.7 \\
This Study & Comb_9,10,6 & 95.3 & 97.6 & 97.8 & 96.1 & $\mathbf{9 6 . 8}$ \\
This Study & Comb_11,10,6 & 96.3 & 96.8 & 97.1 & 96.1 & 96.6 \\
This Study & Comb_12,10,6 & 96.2 & 98.1 & 96.8 & 93.2 & 96.1 \\
\hline
\end{tabular}

TABLE 5

The best results (in percentage \%) achieved for 1189 benchmark (for combinations of feature vectors extracted for attributes number 9,10 , and 12 while $F_{p h}$ and $F_{s}$ are respectively set to 10 and 6 ) compared to the best results reported in the literature.

\begin{tabular}{llccccc}
\hline References & Method & All- $\alpha$ & All- $\beta$ & $\alpha+\beta$ & $\alpha / \beta$ & Overall \\
\hline$[33]$ & Bayes Classifier & 54.8 & 57.1 & 75.2 & 22.2 & 53.8 \\
{$[5]$} & Logistic Regression & 57.0 & 62.9 & 64.7 & 25.3 & 53.9 \\
{$[13]$} & SVM & - & - & - & - & 54.7 \\
{$[53]$} & FKNN & 48.9 & 59.5 & 81.7 & 26.6 & 56.9 \\
{$[19]$} & Stacking Ensemble & - & - & - & - & 58.9 \\
{$[54]$} & WSVM & - & - & - & - & 59.2 \\
{$[48]$} & Specific Tri-peptides & - & - & - & - & 59.9 \\
{$[18]$} & IB1 & 65.3 & 67.7 & 79.9 & 40.7 & 64.7 \\
{$[55]$} & AAD-CGR & 62.3 & 67.7 & 66.5 & 63.1 & 65.2 \\
{$[39]$} & SVM & 75.8 & 75.2 & 82.6 & 31.8 & 67.6 \\
{$[29]$} & AADP-PSSM & 69.1 & 83.7 & 85.6 & 35.7 & 70.7 \\
{$[6]$} & AAC-PSSM-AC & 76.3 & 85.7 & 81.0 & 42.1 & 72.7 \\
This Study & Comb_9,10,6 & 82.1 & 85.1 & 84.8 & 39.4 & 74.3 \\
This Study & Comb_11,10,6 & 80.2 & 83.6 & 85.4 & 44.6 & 74.8 \\
This Study & Comb_12,10,6 & 82.3 & 82.8 & 84.3 & 41.5 & 74.0 \\
\hline
\end{tabular}


TABLE 6

The best results (in percentage \%) achieved for 25PDB benchmark (for combinations of feature vectors extracted for attributes number 9,10 , and 12 while $F_{p h}$ and $F_{s}$ are respectively set to 10 and 6) compared to the best results reported in the literature.

\begin{tabular}{llccccc}
\hline References & Method & All- $\alpha$ & All- $\beta$ & $\alpha+\beta$ & $\alpha / \beta$ & Overall \\
\hline$[5]$ & Logistic Regression & 69.1 & 61.6 & 60.1 & 38.3 & 57.1 \\
{$[48]$} & Specific Tri-peptides & 60.6 & 60.7 & 67.9 & 44.3 & 58.6 \\
{$[19]$} & Stacking Ensemble & - & - & - & - & 59.9 \\
{$[24]$} & LLSC-PRED & 75.2 & 67.5 & 62.1 & 44.0 & 62.2 \\
{$[24]$} & SVM & 77.4 & 66.4 & 61.3 & 45.4 & 62.7 \\
{$[55]$} & AAD-CGR & 64.3 & 65 & 65.0 & 61.7 & 64.0 \\
{$[52]$} & CWT-PCA-SVM & 76.5 & 67.3 & 66.8 & 45.8 & 64.0 \\
{$[29]$} & AADP-PSSM & 83.3 & 78.1 & 76.3 & 54.4 & 72.9 \\
{$[6]$} & AAC-PSSM-AC & 85.2 & 81.3 & 73.7 & 55.2 & 73.9 \\
This Study & Comb_9,10,6 & 85.8 & 81.2 & 79.2 & 59.7 & 76.3 \\
This Study & Comb_11,10,6 & 86.1 & 80.8 & 80.6 & 60.1 & 76.7 \\
This Study & Comb_12,10,6 & 85.2 & 81.4 & 79.1 & 59.1 & 76.0 \\
\hline
\end{tabular}

of $95.7 \%$ reported in the literature for this benchmark [18]. We also achieved up to $96.6 \%$ prediction accuracy using (Comb_11,10,6) up to $0.9 \%$ better than previously reported results for this benchmark.

The proposed method also outperforms similar studies found in the literature for the 1189 and the 25PDB benchmarks. For the 1189 benchmark, we achieve up to $74.8 \%$ prediction accuracy (for Comb_11, 10, 6), 2.1\% better than $72.7 \%$ achieved by reproducing the results of [6] on this benchmark (Table 5). This study also reports $76.7 \%$ prediction accuracy (for Comb_11, 10, 6), $2.8 \%$ better than $73.9 \%$ prediction accuracy achieved by reproducing the results of [6] for the 25PDB benchmark (Table 5).

In order to study the statistical significance of the prediction accuracy enhancement reported in this study, we conduct the paired t-test on our achieved results compared to the highest results reported in the literature. We note that the best prediction results reported on $\mathrm{Z} 277, \mathrm{Z498}, 1189$, and 25PDB benchmarks in the previous studies are $88.8 \%, 95.7 \%, 72.7 \%$, and $73.9 \%$, respectively; while the corresponding results from the present study are $90.3 \%, 96.6 \%, 74.8 \%$, and $76.7 \%$ (using comb_11,10,6 features). For the paired t-test, we get $t$-value equal to 4.484 with 3 degrees of freedom. Our results are statistically better than the best results from the previous studies at $5 \%$ significance level ( $p$ value equals to 0.0103 ).

Achieved results highlight the promising performance of the proposed method to tackle the protein structural class prediction problem compared to the previous studies found in the literature. Beside enhancing the prediction performance for this task, our proposed methods introduce a new approach to explore potential discriminatory information of the physicochemicalbased features in conjunction with the evolutionarybased features which can be used for similar studies.

Exploring results individually for each structural class also shows that for all the employed benchmarks and almost all of the structural classes, proposed method is capable of achieving better results compared to the previously proposed approaches found in the literature (especially the significant enhancement observed for difficult classes $(\alpha / \beta)$ ). It is important to highlight that the reported results are achieved by adding limited number of features to the number of features explored previously [4], [12]. However, despite increasing the number of features, the proposed method provides important information about the impact of the physicochemicalbased attributes for this task. This study also shows the importance of the attributes that have not been explored adequately or completely neglected in previous studies. Achieved results also emphasizes the importance and effectiveness of the proposed feature extraction methods based on the overlapped segmented distribution and autocorrelation concepts to provide more discriminatory information to enhance protein structural class prediction accuracy.

\section{CONCLUSION AND FutURE Works}

In this paper, we have investigated the use of physicochemical-based attributes of the amino acids along with the evolutionary-based information contained in the PSSM for feature extraction. For this, we have selected 15 different physicochemical-based attributes and used each of these attributes to extract two kinds of features: 1) overlapped segmented distribution and 2) overlapped segmented autocorrelation. These features are concatenated with two other kinds of sequential features, PSSM_AAC and PSSM_AC, derived directly from the PSSM.

These features are studied for protein structural class prediction problem using an ensemble of different classifiers on four different benchmarks widely used in the literature. The classification results are reported using the 10 -fold cross validation process. The proposed feature extraction method has been found to perform better than the previously reported results for the protein structural class prediction problem for all the four employed benchmarks [6], [18]. This illustrates the importance of the physicochemical-based attributes (that have not been explored earlier for this task) as well as the overlapped 
segmented-based feature extraction procedure to provide more local and global discriminatory information to tackle the protein structural class prediction problem. For future studies, we aim to explore a wider range of segmented-based feature extraction methods as well as novel weighted-based ensemble of different classifiers. We also aim to collaborate with biological experts to investigate the significant of the explored features in the biological perspective.

\section{APPENDIX \\ Appendix A: The Attribute Selection Procedure}

For a given physicochemical-based attribute, we first extracted six feature groups as follows: Overlapped segmented-based distribution using 10\% distribution factor; Overlapped segmented-based distribution using $5 \%$ distribution factor; Segmented density using 5\% distribution factor (for this feature group, protein sequence is divided into 20 segments with equal length in which each segment is $5 \%$ of total length of protein sequence. Then we calculate density of the explored attribute for each segment (20 features in total). We also add the global density feature to this features to build this feature group (21 features in total)); Overlapped segmentedbased autocorrelation using $25 \%$ distribution factor and $75 \%$ overlapping factor; Overlapped segmented-based autocorrelation using 10\% distribution factor and $70 \%$ overlapping factor; and overlapped segmented based autocorrelation in which the protein sequence is segmented into equal length segments (where each segment is $10 \%$ of the total length of protein sequence. Then segmented autocorrelation is calculated in this feature group in the similar manner that explained in the manuscript using $70 \%$ overlapping factor.).

In the second step, we applied five different classifiers implemented in WEKA namely, Nave Bayes (default parameters adopted), Adaboost.M1 (using 100 base learners and C4.5 as the base learner), Random Forest (using 100 base learners as it was used in [11]), SVM (default parameters are adopted), and KNN (default parameters are adopted) to each extracted feature group individually. Therefore, 30 prediction accuracies were achieved for each individual physicochemical-based attributes (corresponding to each classifier used for six different feature groups $(65=30)$. Finally, we select 15 attributes that attained better results in average for all these 30 prediction accuracies among all 115 physicochemical-based attributes explored in this study.

\section{REFERENCES}

[1] M. Levitt and C. Chothia, "Structural patterns in globular proteins," Nature, vol. 261, no. 5561, pp. 552-558, 1976.

[2] A. G. Murzin, S. E. Brenner, T. Hubbard, and C. Chothia, "Scop: A structural classification of proteins database for the investigation of sequences and structures," Journal of Molecular Biology, vol. 247, no. 4 , pp. 536-540, 1995.
[3] M. Mizianty and L. A. Kurgan, "Modular prediction of protein structural classes from sequences of twilight-zone identity with predicting sequences," BMC Bioinformatics, vol. 10, no. 1, p. 414, 2009.

[4] Z. C. Li, X. B. Zhou, Y. R. Lin, and X. Y. Zou, "Prediction of protein structure class by coupling improved genetic algorithm and support vector machine," Amino Acids, vol. 35, no. 3, pp. 581-590, 2008.

[5] L. A. Kurgan and L. Homaeian, "Prediction of structural classes for protein sequences and domains - impact of prediction algorithms, sequence representation and homology, and test procedures on accuracy," Pattern Recognition, vol. 39, pp. 2323-2343, 2006.

[6] T. Liu, X. Geng, X. Zheng, R. Li, and J. Wang, "Accurate prediction of protein structural class using auto covariance transformation of psi-blast profiles," Amino Acids, vol. 42, pp. 2243-2249, 2012.

[7] L. A. Kurgan, T. Zhang, H. Zhang, S. Shen, and J. Ruan, "Secondary structure-based assignment of the protein structural classes," Amino Acids, vol. 35, pp. 551-564, 2008.

[8] J. Y. Yang, Z. L. Peng, and X. Chen, "Prediction of protein structural classes for low-homology sequences based on predicted secondary structure," BMC Bioinformatics, vol. 11, no. Suppl 1, p. S9, 2010.

[9] K. Y. Feng, Y. D. Cai, and K. C. Chou, "Boosting classifier for predicting protein domain structural class," Biochemical and Biophysical Research Communications, vol. 334, no. 1, pp. 213-217, 2005.

[10] B. Niu, Y. D. Cai, W. C. Lu, G. Z. Li, and K. C. Chou, "Predicting protein structural class with adaboost learner," Protein and Peptide Letters, vol. 13, no. 5, pp. 489-492, 2006.

[11] A. Dehzangi and S. Karamizadeh, "Solving protein fold prediction problem using fusion of heterogeneous classifiers," INFORMATION, An International Interdisciplinary Journal, vol. 14, no. 11, pp. 3611-3622, 2011.

[12] Y. D. Cai, K. Feng, W. Lu, and K. Chou, "Using logitboost classifier to predict protein structural classes," Theoretical Biollogy, vol. 238, pp. 172-176, 2006.

[13] A. Anand, G. Pugalenthi, and P. N. Suganthan, "Predicting protein structural class by svm with class-wise optimized features and decision probabilities," Journal of Theoretical Biology, vol. 253, no. 2, pp. 375-380, 2008.

[14] Y. D. Cai, X. J. Liu, X. B. Xu, and K. C. Chou, "Prediction of protein structural classes by support vector machines," Computers and Chemistry, vol. 26, no. 3, pp. 293-296, 2002.

[15] Y. D. Cai and G. P. Zhou, "Prediction of protein structural classes by neural network," Biochimie, vol. 82, no. 8, pp. 783-785, 2000.

[16] S. Jahandideh, P. Abdolmaleki, M. Jahandideh, and E. B. Asadabadi, "Novel two-stage hybrid neural discriminant model for predicting proteins structural classes," Biophysical Chemistry, vol. 128 , no. 1, pp. 87-93, 2007.

[17] S. Jahandideh, P. Abdolmaleki, M. Jahandideh, and S. H. S. Hayatshahi, "Novel hybrid method for the evaluation of parameters contributing in determination of protein structural classes," Journal of Theoretical Biology, vol. 244, no. 2, pp. 275-281, 2007.

[18] K. Chen, L. A. Kurgan, and J. Ruan, "Prediction of protein structural class using novel evolutionary collocation-based sequence representation," Journal of Computational Chemistry, vol. 29, no. 10, pp. 1596-1604, 2008.

[19] K. D. Kedarisetti, L. A. Kurgan, and S. Dick, "Classifier ensembles for protein structural class prediction with varying homology," Biochemical and Biophysical Research Communications, vol. 348, no. 3, pp. 981-988, 2006.

[20] A. Dehzangi and S. Phon-Amnuaisuk, "Fold prediction problem: The application of new physical and physicochemical- based features," Protein and Peptide Letters, vol. 18, no. 2, pp. 174-185, 2011.

[21] A. Dehzangi, S. Phon-Amnuaisuk, and O. Dehzangi, "Enhancing protein fold prediction accuracy by using ensemble of different classifiers," Australian Journal of Intelligent Information Processing Systems, vol. 26, no. 4, pp. 32-40, 2010.

[22] K. Kavousi, B. Moshiri, M. Sadeghi, B. N. Araabi, and A. A. Moosavi-Movahedi, "A protein fold classifier formed by fusing different modes of pseudo amino acid composition via pssm," Computational Biology and Chemistry, vol. 35, no. 1, pp. 1-9, 2011.

[23] A. Sharma, J. Lyons, A. Dehzangi, and K. K. Paliwal, "A feature extraction technique using bi-gram probabilities of position 
specific scoring matrix for protein fold recognition," Journal of Theoretical Biology, vol. 320, no. 0, pp. 41-46, 2013.

[24] L. A. Kurgan and K. Chen, "Prediction of protein structural class for the twilight zone sequences," Biochemical and Biophysical Research Communications, vol. 357, no. 2, pp. 453-460, 2007.

[25] G. P. Zhou, "An intriguing controversy over protein structural class prediction," Journal of Protein Chemistry, vol. 17, pp. 729-738, 1998.

[26] L. A. Kurgan and L. Homaeian, "Prediction of secondary protein structure content from primary sequence alone - a feature selection based approach," in Machine Learning and Data Mining in Pattern Recognition, 4th International Conference, MLDM, 2005, pp. 334-345.

[27] M. M. Gromiha, "A statistical model for predicting protein folding rates from amino acid sequence with structural class information," Journal of Chemical Information and Modeling, vol. 45, no. 2, pp. 494-501, 2005.

[28] C. Ding and I. Dubchak, "Multi-class protein fold recognition using support vector machines and neural networks," Bioinformatics, vol. 17, pp. 349-358, 2001.

[29] T. Liu and C. Jia, "A high-accuracy protein structural class prediction algorithm using predicted secondary structural information," Journal of Theoretical Biology, vol. 267, no. 3, pp. 272-275, 2010.

[30] S. F. Altschul, T. L. Madden, A. A. Schaffer, J. H. Zhang, Z. Zhang, W. Miller, and D. J. Lipman, "Gapped blast and psi-blast: a new generation of protein database search programs," Nucleic Acids Research, vol. 17, pp. 3389-3402, 1997.

[31] L. A. Kurgan, K. J. Cios, and K. Chen, "Scpred: Accurate prediction of protein structural class for sequences of twilight-zone similarity with predicting sequences," BMC Bioinformatics, vol. 9, p. 226, 2008.

[32] P. Ghanty and N. R. Pal, "Prediction of protein folds: Extraction of new features, dimensionality reduction, and fusion of heterogeneous classifiers," NanoBioscience, IEEE Transactions on, vol. 8, no. 1, pp. 100-110, 2009.

[33] Z. X. Wang and Z. Yuan, "How good is prediction of protein structural class by the component-coupled method?" Proteins: Structure, Function, and Bioinformatics, vol. 38, no. 2, pp. 165-175, 2000.

[34] S. Kawashima, P. P. M. Pokarowska, A. Kolinski, T. Katayama, and M. Kanehisa, "Aaindex: Amino acid index database, progress report," Neucleic Acids, vol. 36, pp. D202-D205, 2008.

[35] V. S. Mathura and D. Kolippakkam, "Apdbase: Amino acid physico-chemical properties database," Bioinformation, vol. 12, no. 1, pp. 2-4, 2005.

[36] Q. Dong, S. Zhou, and G. Guan, "A new taxonomy-based protein fold recognition approach based on autocross-covariance transformation," Bioinformatics, vol. 25, no. 20, pp. 2655-2662, 2009.

[37] X. Zheng, C. Li, and J. Wang, "An information-theoretic approach to the prediction of protein structural class," Journal of Computational Chemistry, vol. 31, no. 6, pp. 1201-1206, 2010.

[38] Y. S. Ding, T. L. Zhang, and K. C. Chou, "Prediction of protein structure classes with pseudo amino acid composition and fuzzy support vector machine network," Protein and Peptide Letters, vol. 14, no. 8, pp. 811-815, 2007.

[39] C. Chen, X. Zhou, Y. Tian, X. Zou, and P. Cai, "Predicting protein structural class with pseudo-amino acid composition and support vector machine fusion network," Analytical Biochemistry, vol. 357, no. 1, pp. 116-121, 2006.

[40] G. I. Webb, "Multiboosting: A technique for combining boosting and wagging," Machine Learning, vol. 40, no. 2, pp. 156-196, 2000.

[41] T. G. Dietterich, "Ensemble methods in machine learning," in International Workshop on Multipe Classifier Systems, 2000, pp. 115.

[42] A. Dehzangi, S. Phon-Amnuaisuk, K. H. Ng, and E. Mohandesi, "Protein fold prediction problem using ensemble of classifiers," in Proceedings of the 16th International Conference on Neural Information Processing: Part II, ser. ICONIP '09, 2009, pp. 503-511.

[43] I. Witten and E. Frank, Data Mining: Practical Machine Learning Tools and Techniques, 2nd ed. Morgan Kaufmann, San Francisco, 2005.

[44] Y. Freund and R. E. Schapire, "Experiments with a new boosting algorithm," in International Conference on Machine Learning, 1996, pp. $148-156$.

[45] P. Jain and J. Hirst, "Automatic structure classification of small proteins using random forest," BMC Bioinformatics, vol. 11, no. 1, p. $364,2010$.
[46] J. Friedman, T. Hastie, and R. Tibshirani, "Additive logistic regression: a statistical view of boosting," Annals of Statistics, vol. 28, pp. 337-407, 2000

[47] V. N. Vapnik, The Nature of Statistical Learning Theory. SpringerVerlag, 1999.

[48] S. Costantini and A. M. Facchiano, "Prediction of the protein structural class by specific peptide frequencies," Biochimie, vol. 91 no. 2, pp. 226-229, 2009.

[49] M. M. Gromiha, "Multiple contact network is a key determinant to protein folding rates," Journal of Chemical Information and Modeling, vol. 49, no. 4, pp. 1130-1135, 2009.

[50] B. Efron and G. Gong, "A leisurely look at the bootstrap, the jackknife, and cross-validation," The American Statistics, vol. 37, no. 1, pp. 36-48, 1983.

[51] Y. D. Cai, X. J. Liu, X. B. Xu, and G. P. Zhou, "Support vector machines for predicting protein structural class," BMC Bioinformatics, vol. 2, no. 1, p. 3, 2001.

[52] Z. C. Li, X. B. Zhou, Z. Dai, and X. Y. Zou, "Prediction of protein structural classes by chou's pseudo amino acid composition: approached using continuous wavelet transform and principal component analysis," Amino Acids, vol. 37, pp. 415-425, 2009.

[53] T. L. Zhang, Y. S. Ding, and K. C. Chou, "Prediction protein structural classes with pseudo amino acid composition: approximate entropy and hydrophobicity pattern," Theoretical Biology, vol. 250, pp. 186-193, 2008

[54] J. D. Qiu, S. H. Luo, J. H. Huang, and R. P. Liang, “Using support vector machines for prediction of protein structural classes based on discrete wavelet transform," Journal of Computational Chemistry, vol. 30, no. 8, pp. 1344-1350, 2009.

[55] J. Y. Yang, Z. L. Peng, Z. G. Yu, R. J. Zhang, V. Anh, and D. Wang, "Prediction of protein structural classes by recurrence quantification analysis based on chaos game representation," Journal of Theoretical Biology, vol. 257, no. 4, pp. 618-626, 2009.

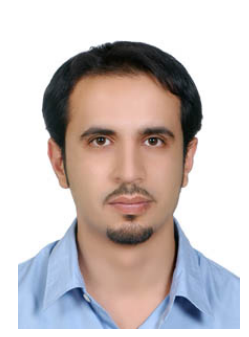

Abdollah Dehzangi received the B.Sc. degree in Computer Engineering-Hardware from Shiraz University, Iran in 2007 and Master degree, in the area of bioinformatics from Multi Media University (MMU), Cyberjaya, Malaysia, in 2011. Since 2011, He is pursuing the Ph.D. degree in Bioinformatics at Griffith University Brisbane, Australia. He is also a researcher in National ICT Australia (NICTA). His research interests include Bioinformatics, protein fold and structural class prediction problems, data mining, statistical learning theory, and pattern recognition. 


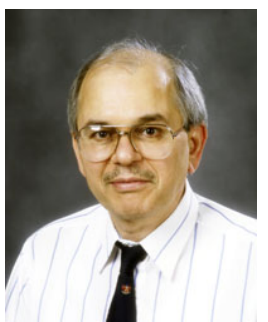

Kuldip Paliwal received the B.S. degree from Agra University, Agra, India, in 1969, the M.S. degree from Aligarh Muslim University, Aligarh, India, in 1971 and the Ph.D. degree from Bombay University, Bombay, India, in 1978. He has been carrying out research in the area of speech processing since 1972. He has worked at a number of organizations including Tata Institute of Fundamental Research, Bombay, India, Norwegian Institute of Technology, Trondheim, Norway, University of Keele, U.K., AT \& T Bell Laboratories, Murray Hill, New Jersey, U.S.A., AT \& T Shannon Laboratories, Florham Park, New Jersey, U.S.A., and Advanced Telecommunication Research Laboratories, Kyoto, Japan. Since July 1993, he has been a professor at Griffith University, Brisbane, Australia, in the School of Micro electronic Engineering. His current research interests include speech recognition, speech coding, speaker recognition, speech enhancement, face recognition, image coding, bioinformatics, protein fold and structural class prediction problems, pattern recognition and artificial neura networks. He has published more than 300 papers in these research areas. Dr. Paliwal is a Fellow of Acoustical Society of India. He has served the IEEE Signal Processing Societys Neural Networks Technical Committee as a founding member from 1991 to 1995 and the Speech Processing Technical Committee from 1999 to 2003. He was an Associate Editor of the IEEE Transactions on Speech and Audio Processing during the periods 1994-1997 and 2003-2004. He also served as Associate Editor of the IEEE Signal Processing Letters from 1997 to 2000. He was the editor-in-chief of Speech Communication Journal from 2005 to 2011. He was the General Co-Chair of the Tenth IEEE Workshop on Neural Networks for Signal Processing (NNSP2000).

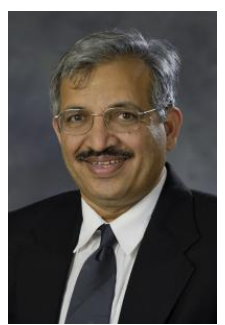

Abdul Sattar holds a BSc (Physics, Chemistry and Mathematics) and an MSc (Physics) from the University of Rajasthan, India, in 1977, and 1979 an MPhil in Computer and Systems Sciences from the Jawaharlal Nehru University, India, and an MMath in Computer Science from the University of Waterloo, Canada, and a PhD in Computer Science (with specialization in Artificial Intelligence) from the University of Alberta, Canada, in 1990. He is the founding Director of the Institute for Integrated and Intelligent Systems and a Professor of Computer Science and Artificial Intelligence at Griffith University. He is also a Research Leader at National ICT Australia (NICTA) Queensland Research Lab (QRL), where he has held the positions of QRL Education Director (2006-08) and Leader of the Smart Applications For Emergencies (SAFE) project (2005-08), and is currently leading the QRL node of NICTA's largest project, Advanced Technologies for Optimization and Modelling In Constraints (ATOMIC). $\mathrm{He}$ has been an academic staff member at Griffith University since February 1992 as a lecturer (1992-95), senior lecturer (1996-99), and professor (2000-present) within the School of Information and Communication Technology. Prior to his career at Griffith University, he was a lecturer in Physics in Rajasthan, India (1980-82), and a research scholar at Jawaharlal Nehru University, India (1982-85), the University of Waterloo, Canada (1985-87), and the University of Alberta, Canada (19871991). His research interests include knowledge representation and reasoning, constraint satisfaction, intelligent scheduling, rational agents, propositional satisfiability, temporal reasoning, temporal databases, and bioinformatics.

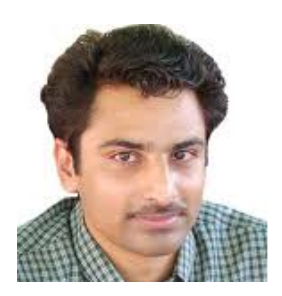

Alok Sharma received the BTech degree from the University of the South Pacific (USP), Suva, Fiji, in 2000 and the MEng degree, with an academic excellence award, and the $\mathrm{PhD}$ degree in the area of pattern recognition from Griffith University, Brisbane, Australia, in 2001 and 2006, respectively. He was with the University of Tokyo (2010-2012) as a research fellow. He is also with the Signal Processing Laboratory, Griffith University, and the University of the South Pacific. He participated in various projects carried out in conjunction with Motorola (Sydney), Auslog Pty., Ltd. (Brisbane), CRC Micro Technology (Brisbane), the French Embassy (Suva) and JSPS (Japan). His research interests include pattern recognition, computer security, human cancer classification and protein fold and structural class prediction problems. He reviewed several articles and is in the editorial board of several journals. He is a member of IEEE.

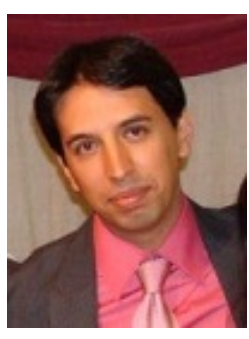

Omid Dehzangi received his B.Eng and M.Eng degrees in computer engineering from Shiraz University, Iran, 2004 and 2007. He received his Ph.D. degree in computer engineering at Nanyang Technological University, Singapore 2012 . He is currently working as a postdoctoral researcher in Electrical Engineering Department, University of Texas at Dallas. His research interests include pattern recognition, brain-computer interface, speech and signal processing, bioinformatics, protein fold and structural class prediction problems, and discriminative analysis. 